\title{
INHALT
}

Hauptaufsätze

Seite

E. Fehlberg: Eine Runge-Kutta-Nyström-Formel 9-ter Ordnung mit Schrittweitenkontrolle für Differentialgleichungen $\ddot{x}=f(t, x) \ldots \ldots \ldots \ldots \ldots$. . . . . . . . . . . . . . . . . . . . . . . . . .

G. Lippold: Schätzung von Diskretisierungsfehlern bei der Lösung von Gleichungen in Banach-Räumen . . . . 487

M. A. Jiménez Pozo: Convergence of Sequences of Linear Functionals . . . . . . . . . . . . . . . . 495

W. Kampowsky: Optimalitätsbedingungen für Prozesse in Evolutionsgleichungen 1. Ordnung . . . . . . . . 501

K. Gröger / R. Hünlich: On the Theory of Diffusion Processes in Viscoelastic Media . . . . . . . . . . . 513

Kleine Mitteilungen

A. J. Prasad / N. R. Rajappa: Nonsteady Three-Dimensional Stagnation Point Flow with Hard Blowing . . . 521

H. F. Baver: On the Numerical Value of the Roots of the Associated Legendre Function with Respect to the

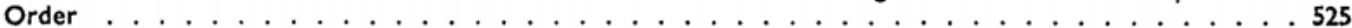

A. D. Rawlins: The Explicit Wiener-Hopf Factorisation of a Special Matrix . . . . . . . . . . . . . . . . . . . 527

W. Grecksch: Steuerung zufälliger Felder auf der Grundlage eines Satzes vom Girsanov-Typ. . . . . . . . . 528

R. Grübel / U. Jensen: On the Moments of the Number of Renewal Epochs . . . . . . . . . . . . . . . . 531

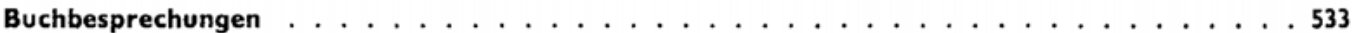

Eingegangene Bücher $\ldots \ldots \ldots \ldots \ldots \ldots \ldots$

Nachrichten . . . . . . . . . . . . . . . . . . . . . 539

Wir biften, Manuskriptsendungen zweifach (Original und eine Kopie, sprachlich einwandfrei, Formeln mit Maschine oder in Druckschrift geschrieben) an folgende Anschrift zu richten:

Zeitschrift für Angewandte Mathematik und Mechanik,

Institut für Mechanik der Akademie der Wissenschaften der DDR

DDR-1199 Berlin, Rudower Chaussee 5.

$\mathrm{Zu}$ den Arbeiten, die als Hauptaufsätze bestimmt sind, ist auf gesondertem Blatt eine Zusammenfassung von $\mathbf{5}$ bis 10 Zeilen in englischer und (möglichst) deutscher und russischer Sprache belzufügen. Ausführliche Hinweise für die Autoren, um deren strikte Berücksichtigung gebeten wird, finden sich Im Anschluß an das Inhaltsverzeichnis des Jahrganges 60 (1980).

Die Autoren erhalten von den Hauptaufsätzen 75, von den Kleinen Mitteilungen 25 Sonderdrucke ohne Berechnung, darüber hinaus weitere Sonderdrucke gegen Berechnung.

Der Verlag behält sich für alle Beiträge das Recht der Vervielfältigung und Ubersetzung vor.

Bestellungen sind zu richten

- in der DDR an den Postzeitungsvertrieb unter Angabe der Kundennummer des Bestellenden oder an den AKADEMIE-VERLAG, DDR-1080 Berlin, Leipziger Straße 3-4

- im sozialistischen Ausiand an eine Buchhandiung für fremdsprachige Literatur oder an den zuständigen Postzeifungsvertrieb

- in der BRD und Berlin(West) an eine Buchhandlung oder an die Auslieferungsstelle KUNST UND WISSEN, Erich Bleber OHG, D-7000 Stuttgart 1, Wilhelmstraße 4-6

- in den übrigen westeuropäischen Ländern an eine Buchhandlung oder an die Auslieferungssfelle KUNST UND WISSEN, Erich Bieber GmbH, Ch-8008 Zürich/Schweiz, Dufourstraße 51

- im übrigen Ausland an den Internationalen Buch- und Zeitschriftenhandel; den Buchexport, Volkseigener Außenhandelsbetrieb der Deutschen Demokratischen Republik, DDR-7010 Leipzig, Postfach 160; oder an den AKADEMIE-VERLAG, DDR-1080 Berlin, Leipziger Straße 3-4

\section{ZEITSCHRIFT FÜR ANGEWANDTE MATHEMATIK UND MECHANIK}

Herausgeber und Chefredakteur: Prof. Dr. Günter Schmidt. Redaktion: Dr. Winfried Heinrich, Dr. Horst Weinert, Dipl.-Math. Friedhild Dudel, Helga Rühl, Institut für Mechanik der Akademie der Wissenschaften der DDR. Verlag: Akademie-Verlag, DDR-1080 Berlin, Leipziger Straße 3-4; Fernruf: 2236221 oder 2236221. Telex-Nr.: 114420; Bank: Staatsbank der DDR, Berlin, Kto.-Nr.: 6836-26-20712. Anschrift der Redaktion: Instituł für Mechanik der Akademie der Wissenschaften, DDR-1199 Berlin, Rudower Chaussee 5, Fernruf: 6702841.

Veröffeatlicht unter der Lizenznummer 1282 des Presseamtes beim Vorsitzenden des Ministerrates der Deutschen Demokratischen Republik. Gesamtherstellung: VEB Druckerei .Thomas Müntzer". DDR-5820 Bad Langensalza. Erscheinungsweise: Die Zoitschrift für Angewandte Mathematik und Mechanik erscheint monatlich. Die 12 Heffe eines Jahres einschlieBlich Tagungshefte bilden einen Band. Bezugspreis io Band $360,-$ M zuzuglich versan

(c) 1981 by Akademie-Verlag Berlin . Printed in the German Democratic Ropublic.

AN (EDV) 35937 